\title{
Risk-Controlled Multiobjective Portfolio Selection Problem Using a Principle of Compromise
}

\author{
Takashi Hasuike $^{1}$ and Hideki Katagiri ${ }^{2}$ \\ ${ }^{1}$ Graduate School of Information Science and Technology, Osaka University, 2-1 Yamadaoka, Suita, Osaka 565-0871, Japan \\ ${ }^{2}$ Graduate School of Engineering, Hiroshima University, 1-4-1 Kagamiyama, Higashi-Hiroshima, Hiroshima 739-8527, Japan \\ Correspondence should be addressed to Takashi Hasuike; thasuike@ist.osaka-u.ac.jp
}

Received 28 February 2014; Accepted 9 April 2014; Published 29 April 2014

Academic Editor: Pankaj Gupta

Copyright (c) 2014 T. Hasuike and H. Katagiri. This is an open access article distributed under the Creative Commons Attribution License, which permits unrestricted use, distribution, and reproduction in any medium, provided the original work is properly cited.

\begin{abstract}
This paper proposes a multiobjective portfolio selection problem with most probable random distribution derived from current market data and other random distributions of boom and recession under the risk-controlled parameters determined by an investor. The current market data and information include not only historical data but also interpretations of economists' oral and linguistic information, and hence, the boom and recession are often caused by these nonnumeric data. Therefore, investors need to consider several situations from most probable condition to boom and recession and to avoid the risk less than the target return in each situation. Furthermore, it is generally difficult to set random distributions of these cases exactly. Therefore, a robust-based approach for portfolio selection problems using the only mean values and variances of securities is proposed as a multiobjective programming problem. In addition, an exact algorithm is developed to obtain an explicit optimal portfolio using a principle of compromise.
\end{abstract}

\section{Introduction}

Portfolio selection problem has been important in applied finance and investment science to obtain the optimal asset allocation among securities since Markowitz proposed mean variance model (Markowitz [1]). Many portfolio models based on mathematical programming were developed after mean variance model, for instance, mean-absolute-deviation model (Konno et al. $[2,3]$ ), semivariance model (Bawa and Lindenberg [4]), safety-first model (Elton and Gruber [5]), value-at-risk (VaR), and conditional value-at-risk (cVaR) model (Rockafellar and Uryasev [6]).

Standard portfolio models were formulated as stochastic programming problem with random future returns. On the other hand, current market data is often imperfect and ambiguous, and the investor also interprets economist's oral and linguistic information subjectively. Therefore, it is almost impossible to determine random distributions of future returns. As a nonprobabilistic approach, many researchers proposed fuzzy-based portfolio selection problems using the fuzzy theory (Bilbao-Terol et al. [7], Carlsson et al. [8], Duan and Stahlecker [9], Huang [10, 11], Inuiguchi and Tanino [12],
Tanaka et al. $[13,14]$, and Watada [15]). Furthermore, some researchers proposed portfolio models with both randomness and fuzziness, for instance, fuzzy random portfolio selection problems (Katagiri et al. [16]) and random fuzzy portfolio selection problems (Hasuike et al. [17], Huang [18]). Hasuike and Ishii [19] proposed a portfolio model with some possibility of the expected returns. In this model, they assumed that current market data and information included not only historical data but also interpretations of economists' oral and linguistic information, and the boom and recession are often caused by these nonnumeric data. Thus, it is important to set future returns considering most probable random distribution derived from current market data and other random distributions of boom and recession, and hence, we formulate a multiobjective portfolio selection problem with several possible future returns.

As another portfolio model under uncertainty, some researchers recently deal with an uncertainty set of future returns, which is generally formulated as box or ellipsoidal sets, not assuming certain random distributions and membership functions, and robust portfolio selection problems with the uncertainty set have been actively studied. It is 
important for investors to avoid the conceivable worst case and to decide on an optimal investment strategy using the robust approach (e.g., Goldfarb and Iyengar [20], Gregory et al. [21], Liesiö et al. [22], and Lobo [23]). Robust optimization problems have become an active research area in mathematical programming, leading to various studies of solution algorithms (e.g., Ben-Tal and Nemirovski [24, 25]). However, investor must exactly determine the explicit uncertainty set of future returns in order to obtain an optimal portfolio in mathematical programming. The idea of robustness is useful and important to avoid the conceivable worst case of total return loss in risk management. In addition, investors can easily obtain the mean values and variances of securities to collect current numerical market data and undertake the statistical analysis. Hasuike and Katagiri [26] proposed an interactive and theoretical approach for robust portfolio selection problem. In this paper, we also apply the idea of robustness using the mean values and variances of securities to a multiobjective portfolio selection problem considering several situations.

This paper is organized as follows. In Section 2, we introduce mathematical formulation of existing robust portfolio selection problems concept. In Section 3, we propose a multiobjective portfolio selection problem using the robustbased portfolio model shown in Section 2. It is hard to solve this proposed problem directly since a complete optimal solution that simultaneously optimizes all of the multiobjective functions does not always exist. Therefore, we adopt a principle of compromise considering the shortest distance from positive ideal values, called Minkowski's $L p$-metric, in order to solve the proposed problem in mathematical programming. In addition, we develop an exact algorithm to obtain an explicit optimal portfolio of the proposed problem. In Section 4, we conclude this paper.

\section{Mathematical Formulation of a Robust-Based Portfolio Selection Problem}

A simple objective of portfolio selection problems is generally maximizing the total profit to decide an asset allocation rate of each security. The mathematical formulation is introduced as follows:

$$
\begin{aligned}
& \text { Maximize } \mathbf{r}^{t} \mathbf{x} \\
& \text { subject to } \mathbf{1}^{t} \mathbf{x}=1, \quad \mathbf{x} \geq \mathbf{0},
\end{aligned}
$$

where the notation means as follows: $\mathbf{r}$ is the future return column vector of securities, $\mathbf{r} \in \mathbf{R}^{n}, \mathbf{x}$ is the portfolio rate column vector of securities, $\mathbf{x} \in \mathbf{R}^{n}$, and $n$ is the total number of securities.

It is generally impossible to determine future returns as constant values in the real market, due to difficulty of return forecast derived from economic fluctuations. Furthermore, in standard academic portfolio models, the normal distribution is assumed as the random distribution of each future return. However, it is difficult to set the specific random distribution exactly in the real market. On the other hand, investors can collect historical data of each security in the current market and calculate the mean value and variance statistically. Therefore, we need to develop a portfolio model using only mean values and variances of securities without assuming the specific random distributions.

Furthermore, most investors also tend to avoid some risks such as loss of the total return holding certain level. Particularly, it is important to do the risk-averse decision due to the high possibility of loss under uncertainty of future returns. Therefore, in risk management to avoid a greater loss of the total return as much as possible, we apply a robust programming approach to the proposed portfolio model.

2.1. Uncertainty Sets. A general ellipsoidal uncertainty set of future returns $U_{r}$ is given as the following constraint:

$$
U_{r}=\{\mathbf{r} \mid \mathbf{r}=\overline{\mathbf{r}}+\mathbf{Q} \mathbf{w},\|\mathbf{w}\| \leq d\}, \quad\left(\|\mathbf{w}\|=\sqrt{\sum_{j=1}^{n} w_{j}^{2}}\right),
$$

where $\overline{\mathbf{r}}$ is the column vector of center values for $\mathbf{r}$ and $\mathbf{Q}$ is a regular matrix, which represents the relation between any two securities. In (2), we set a positive value $d$ as a degree of considerable range. In general robust programming optimization, parameter $d$ is often set as 1 . In (2), we set parameters $\mathbf{w}^{\prime}=\mathbf{w} / d, \mathbf{Q}^{\prime}=d \mathbf{Q}$ which equivalently transform into the following uncertainty set:

$$
U_{r}=\left\{\mathbf{r} \mid \mathbf{r}=\overline{\mathbf{r}}+\mathbf{Q}^{\prime} \mathbf{w}^{\prime},\left\|\mathbf{w}^{\prime}\right\| \leq 1\right\}
$$

We may verify that the worst case of total return $\mathbf{r}^{t} \mathbf{x}$ is attained when $\mathbf{w}^{\prime}=-\mathbf{Q}^{\prime} \mathbf{x} /\left\|\mathbf{Q}^{\prime} \mathbf{x}\right\|$; that is, $\mathbf{w}=-d \mathbf{Q}^{\prime} \mathbf{x} /\left\|\mathbf{Q}^{\prime} \mathbf{x}\right\|$. Therefore, the worst total return $\min _{\mathbf{r} \in U_{r}} \mathbf{r}^{t} \mathbf{x}$ under the maximization of the total return is obtained as follows:

$$
\begin{aligned}
\min _{\mathbf{r} \in U_{r}} \mathbf{r}^{t} \mathbf{x} & =\min _{\|\mathbf{w}\| \leq d}(\overline{\mathbf{r}}+\mathbf{Q} \mathbf{w})^{t} \mathbf{x} \\
& =\overline{\mathbf{r}}^{t} \mathbf{x}-d\left(\frac{(\mathbf{Q} \mathbf{x})^{t} \mathbf{Q} \mathbf{x}}{\|\mathbf{Q} \mathbf{x}\|}\right)=\overline{\mathbf{r}}^{t} \mathbf{x}-d\|\mathbf{Q} \mathbf{x}\| .
\end{aligned}
$$

This formula can represent robustness by adjusting parameter $d$ according to investor's satisfaction, and, hence, parameter $d$ is regarded as a robustness parameter. If the investor is riskaverse, she or he will set a larger value of $d$, and if not, she or he will set a smaller value of $d$. Therefore, using formula (4) for the total return, we propose a robust-based portfolio selection problem maximizing robustness parameter $d$ as well as maximizing the total return.

2.2. Formulation of Our Proposed Robust-Based Model. We introduce the following parameters setting variance-covariance matrix $\mathbf{V}$ where $\sigma_{i j}$ is the $i j$ th component of $\mathbf{V}$ :

$$
E\left(\mathbf{r}^{t} \mathbf{x}\right)=\overline{\mathbf{r}}^{t} \mathbf{x}, \quad \mathbf{V}\left(\mathbf{r}^{t} \mathbf{x}\right)=\mathbf{x}^{t} \mathbf{V} \mathbf{x}
$$

From current numerical market data, $E\left(\mathbf{r}^{t} \mathbf{x}\right)$ and $\mathbf{V}\left(\mathbf{r}^{t} \mathbf{x}\right)$ can be calculated. Using $E\left(\mathbf{r}^{t} \mathbf{x}\right)$ and $\mathbf{V}\left(\mathbf{r}^{t} \mathbf{x}\right)$, we formulate a portfolio selection problem with robustness parameter $d(>0)$ based on the idea of robust optimization (4). If the value of robustness parameter $d$ is larger, the assumed area of uncertainty set is also wide, and it urges the investor to deal 
with more robust cases than the small value of $d$. Therefore, the following robust-based portfolio selection problem is formulated by setting robustness parameter $d$ based on the investor's risk-averse subjectivity:

$$
\begin{gathered}
\text { Maximize } E\left(\mathbf{r}^{t} \mathbf{x}\right)-d \sqrt{\mathbf{V}\left(\mathbf{r}^{t} \mathbf{x}\right)} \\
\text { subject to } E\left(\mathbf{r}^{t} \mathbf{x}\right) \geq f, \\
\mathbf{1}^{t} \mathbf{x}=1, \quad \mathbf{x} \geq \mathbf{0},
\end{gathered}
$$

where $f$ is the standard target value of total future profit according to the investor's demand. With respect to the objective function, if future returns occur according to normal distributions and robust parameter $d$ is set as $\beta$-quantile point of normal distribution, and $d$-sigma value can be regarded as the value-at-risk point of normal distribution under probability level $\beta$. Therefore, the investor can obtain the total return more than $E\left(\mathbf{r}^{t} \mathbf{x}\right)$ avoiding the risk by maximizing $E\left(\mathbf{r}^{t} \mathbf{x}\right)-$ $d \sqrt{\mathbf{V}\left(\mathbf{r}^{t} \mathbf{x}\right)}$. In this paper, we call objective function $E\left(\mathbf{r}^{t} \mathbf{x}\right)-$ $d \sqrt{\mathbf{V}\left(\mathbf{r}^{t} \mathbf{x}\right)} d$-sigma value. Problem (6) is a convex programming problem, and hence, we obtain the optimal solution using the convex programming approach.

\section{Our Proposed Model Robust-Based Multiobjective Portfolio Selection Problem}

In practical investment situations, it is natural for the investor to consider maximizing the target total profit as well as minimizing the total risk, that is, maximizing the robustness parameter in risk management. Therefore, problem (6) is reformulated as the following problem:

$$
\begin{array}{ll}
\text { Maximize } & E\left(\mathbf{r}^{t} \mathbf{x}\right)-d \sqrt{\mathbf{V}\left(\mathbf{r}^{t} \mathbf{x}\right)} \\
\text { Maximize } & E\left(\mathbf{r}^{t} \mathbf{x}\right) \\
\text { subject to } & \mathbf{1}^{t} \mathbf{x}=1, \quad \mathbf{x} \geq \mathbf{0} .
\end{array}
$$

Furthermore, it often occurs that a future economy becomes boom or recession than envisioned values derived from the historical analysis based on current market data. As well as expected value $\overline{\mathbf{r}}_{C}$ with the variance-covariance matrix $\mathbf{V}_{C}$ in the envisioned future market, it is natural that investors set expected return $\overline{\mathbf{r}}_{U}$ with the variance-covariance matrix $\mathbf{V}_{U}$ in the boom and expected return $\overline{\mathbf{r}}_{L}$ with the variance-covariance matrix $\mathbf{V}_{L}$ in the recession, respectively. These cases generally have larger uncertainty than usual, and, hence, $\sigma_{L j}^{2}>$ $\sigma_{C j}^{2}$ and $\sigma_{U j}^{2}>\sigma_{C j}^{2}$ are assumed. We propose the following multiobjective portfolio selection problem based on problem (7):

$$
\begin{array}{ll}
\text { Maximize } & E\left(\overline{\mathbf{r}}_{C}^{t} \mathbf{x}\right)-d_{C} \sqrt{\mathbf{V}\left(\overline{\mathbf{r}}_{C}^{t} \mathbf{x}\right)} \\
\text { Maximize } & E\left(\overline{\mathbf{r}}_{U}^{t} \mathbf{x}\right)-d_{U} \sqrt{\mathbf{V}\left(\overline{\mathbf{r}}_{U}^{t} \mathbf{x}\right)} \\
\text { Maximize } & E\left(\overline{\mathbf{r}}_{L}^{t} \mathbf{x}\right)-d_{L} \sqrt{\mathbf{V}\left(\overline{\mathbf{r}}_{L}^{t} \mathbf{x}\right)}
\end{array}
$$

$$
\begin{array}{ll}
\text { Maximize } & E\left(\overline{\mathbf{r}}_{C}^{t} \mathbf{x}\right) \\
\text { Maximize } & E\left(\overline{\mathbf{r}}_{U}^{t} \mathbf{x}\right) \\
\text { Maximize } & E\left(\overline{\mathbf{r}}_{L}^{t} \mathbf{x}\right) \\
\text { subject to } & \mathbf{1}^{t} \mathbf{x}=1, \quad \mathbf{x} \geq \mathbf{0} .
\end{array}
$$

We set $z_{d_{k}}=E\left(\overline{\mathbf{r}}_{k}^{t} \mathbf{x}\right)-d_{k} \sqrt{\mathbf{V}\left(\overline{\mathbf{r}}_{k}^{t} \mathbf{x}\right)}, k \in\{C, L, U\}$. It is hard to solve this problem directly since a complete optimal solution that simultaneously optimizes all of the multiobjective functions does not always exist, and hence, various approaches to obtain a compromise solution for the multiobjective programming problem have been proposed. In this paper, we adopt a principle of compromise considering the shortest distance from positive ideal values $z_{d_{k}}^{*}$ and $E^{*}\left(\overline{\mathbf{r}}_{k}^{t} \mathbf{x}\right)$ and the farthest distance from minimum target values $z_{d_{k}}^{-}$and $E^{-}\left(\overline{\mathbf{r}}_{k}^{t} \mathbf{x}\right)$, called Minkowski's Lp-metric. Minkowski's Lpmetric is defined by the following form using $L p$-norm $(p>$ $1)$ :

$$
\begin{aligned}
Z_{p}(\mathbf{x})= & \left\{\sum_{k \in\{C, L, U\}} w_{d_{k}}\left\{\frac{z_{d_{k}}^{*}-z_{d_{k}}}{z_{d_{k}}^{*}-z_{d_{k}}^{-}}\right\}^{p}\right. \\
& \left.+\sum_{k \in\{C, L, U\}} w_{E_{k}}\left\{\frac{E^{*}\left(\overline{\mathbf{r}}_{k}^{t} \mathbf{x}\right)-E\left(\overline{\mathbf{r}}_{k}^{t} \mathbf{x}\right)}{E^{*}\left(\overline{\mathbf{r}}_{k}^{t} \mathbf{x}\right)-E^{-}\left(\overline{\mathbf{r}}_{k}^{t} \mathbf{x}\right)}\right\}^{p}\right\}^{1 / p},
\end{aligned}
$$

where $w_{d_{k}}$ and $w_{E_{k}},(k \in\{C, L, U\})$ are the relative importance of each objective function and $p$ is the parameter of norm functions. To simplify the discussion, we set $p=1$ in this paper. The case $p=1$ is operationally and practically important, which provides better credibility than others and emphasizes the sum of individual distances in the utility concept [10]. Main problem (8) is transformed into the following single-objective programming problem:

$$
\begin{array}{ll}
\text { Minimize } & Z_{p}(\mathbf{x}) \\
\text { subject to } & \mathbf{1}^{t} \mathbf{x}=1, \quad \mathbf{x} \geq \mathbf{0} .
\end{array}
$$

That is,

$$
\begin{aligned}
\text { Minimize } & \sum_{k \in\{C, L, U\}} w_{d_{k}}\left\{\frac{z_{d_{k}}^{*}-z_{d_{k}}}{z_{d_{k}}^{*}-z_{d_{k}}^{-}}\right\} \\
& +\sum_{k \in\{C, L, U\}} w_{E_{k}}\left\{\frac{E^{*}\left(\overline{\mathbf{r}}_{k}^{t} \mathbf{x}\right)-E\left(\overline{\mathbf{r}}_{k}^{t} \mathbf{x}\right)}{E^{*}\left(\overline{\mathbf{r}}_{k}^{t} \mathbf{x}\right)-E^{-}\left(\overline{\mathbf{r}}_{k}^{t} \mathbf{x}\right)}\right\}
\end{aligned}
$$

subject to $\quad \mathbf{1}^{t} \mathbf{x}=1, \quad \mathbf{x} \geq \mathbf{0}$.

In order to solve this problem, investors need to set parameters $z_{d_{k}}^{*}, z_{d_{k}}^{-}, E^{*}\left(\overline{\mathbf{r}}_{k}^{t} \mathbf{x}\right)$, and $E^{-}\left(\overline{\mathbf{r}}_{k}^{t} \mathbf{x}\right),(k \in\{C, L, U\})$ explicitly. Therefore, we develop an exact algorithm to obtain optimal values of $z_{d_{k}}^{*}, z_{d_{k}}^{-}, E^{*}\left(\overline{\mathbf{r}}_{k}^{t} \mathbf{x}\right)$, and $E^{-}\left(\overline{\mathbf{r}}_{k}^{t} \mathbf{x}\right),(k \in\{C, L, U\})$. 
3.1. Subproblems of Our Proposed Model and the Exact Algorithm. Subproblems to obtain positive ideal value $z_{d_{k}}^{*}$ and $E^{*}\left(\overline{\mathbf{r}}_{k}^{t} \mathbf{x}\right)$ are as follows:

$$
\begin{array}{ll}
\text { Maximize } & E\left(\overline{\mathbf{r}}_{k}^{t} \mathbf{x}\right)-d_{k} \sqrt{\mathbf{V}\left(\overline{\mathbf{r}}_{k}^{t} \mathbf{x}\right)} \\
\text { subject to } & \mathbf{1}^{t} \mathbf{x}=1, \quad \mathbf{x} \geq \mathbf{0}, \\
& (k \in\{C, L, U\}), \\
\text { Maximize } & E\left(\overline{\mathbf{r}}_{k}^{t} \mathbf{x}\right) \\
\text { subject to } & \mathbf{1}^{t} \mathbf{x}=1, \quad \mathbf{x} \geq \mathbf{0}, \\
& (k \in\{C, L, U\}) .
\end{array}
$$

In this paper, the minimum target values $z_{d_{k}}^{-}$and $E^{-}\left(\overline{\mathbf{r}}_{k}^{t} \mathbf{x}\right)$ of objective functions are also set by the investor. Since problem (13) is a linear knapsack problem, the optimal portfolio and optimal value are easily and explicitly obtained as follows: (optimal portfolio and optimal value of problem (13))

$$
\begin{gathered}
x_{i}^{*}= \begin{cases}1, & i=\arg \max _{1 \leq j \leq n}\left\{\bar{r}_{k j}\right\} \\
0, & \text { otherwise }\end{cases} \\
E^{*}\left(\overline{\mathbf{r}}_{k}^{t} \mathbf{x}\right)=\max _{1 \leq j \leq n}\left\{\bar{r}_{k j}\right\}, \\
(k \in\{C, L, U\}) .
\end{gathered}
$$

We consider an exact algorithm to obtain each optimal portfolio of problem (12). Since $\mathbf{V}_{k}$ in problem (12) is a positive definite matrix, we perform the following equivalent transformations of variables:

$$
\begin{aligned}
& \mathbf{x}^{t} \mathbf{V}_{k} \mathbf{x}=\mathbf{y}_{k}^{t} \mathbf{y}_{k} \\
& \mathbf{V}_{k}=\mathbf{P}_{k}^{t} \boldsymbol{\Lambda}_{k} \mathbf{P}_{k}, \quad\left(\mathbf{P}_{k}:\right. \text { unitary matrix consisting } \\
& \text { of eigen vector of } \mathbf{V}_{k} \text { ) } \\
& \boldsymbol{\Lambda}_{k}=\left(\begin{array}{ccc}
\lambda_{k 1} & & \mathbf{0} \\
& \ddots & \\
\mathbf{0} & & \lambda_{k n}
\end{array}\right), \quad\left(\lambda_{k i}: \text { eigen value of } \mathbf{V}_{k}\right) \\
& \boldsymbol{\Lambda}_{k}=\left(\Lambda_{k}^{1 / 2}\right)^{t} \Lambda_{k}^{1 / 2}, \quad \boldsymbol{\Lambda}_{k}^{1 / 2}=\left(\Lambda_{k}^{1 / 2}\right)^{t}=\left(\begin{array}{ccc}
\sqrt{\lambda_{k 1}} & & \mathbf{0} \\
& \ddots & \\
\mathbf{0} & & \sqrt{\lambda_{k n}}
\end{array}\right) \\
& \mathbf{y}_{k}=\Lambda_{k}^{1 / 2} \mathbf{P}_{k} \mathbf{x}, \quad \overline{\mathbf{r}}_{\mathbf{k}}^{\prime}=\left(\Lambda_{k}^{1 / 2}\right)^{-1} \mathbf{P}_{k} \overline{\mathbf{r}}_{k}, \\
& \mathbf{a}_{k}=\left(\Lambda_{k}^{1 / 2}\right)^{-1} \mathbf{P}_{k} \mathbf{1}
\end{aligned}
$$

Using these transformations of variables, problem (12) is equivalently transformed into the following problem:

$$
\begin{array}{ll}
\text { Minimize } & -\overline{\mathbf{r}}_{\mathbf{k}}^{\prime} \mathbf{y}_{k}+d_{k} \sqrt{\mathbf{y}_{k}^{t} \mathbf{y}_{k}} \\
\text { subject to } & \mathbf{a}_{k}^{t} \mathbf{y}_{k}=1, \quad \mathbf{y}_{k} \geq \mathbf{0} .
\end{array}
$$

This problem still includes square root term $\sqrt{\mathbf{y}_{k}^{t} \mathbf{y}_{k}}$. Therefore, in order to obtain the exact optimal portfolio of problem (16), we introduce the following auxiliary problem $P^{R_{k}}$ using a parameter $R_{k}$ not including the square root term:

$$
\begin{array}{ll}
\text { Minimize } & R_{k}\left(-\overline{\mathbf{r}}_{\mathbf{k}}^{\prime} \mathbf{y}_{k}\right)+\frac{d_{k}}{2}\left(\mathbf{y}_{k}^{t} \mathbf{y}_{k}\right) \\
\text { subject to } & \mathbf{a}_{k}^{t} \mathbf{y}_{k}=1, \quad \mathbf{y}_{k} \geq \mathbf{0} .
\end{array}
$$

Since this problem is a quadratic convex programming problem, we solve problem (17) more easily than problem (16). Furthermore, with respect to the relation between problem (16) and its auxiliary problem (17), the following theorem holds based on Hasuike and Katagiri [26].

Theorem 1 (see [26]). Let $\mathbf{y}_{k}^{\left(R_{k}\right)}$ be the optimal solution of auxiliary problem (17). If $R_{k}=\sqrt{\sum_{j=1}^{n}\left\{y_{k j}^{\left(R_{k}\right)}\right\}^{2}}$ holds, $\mathbf{y}_{k}^{\left(R_{k}\right)}$ is also the optimal solution of problem (16).

This theorem means that we can obtain the optimal solution of problem (16) by solving the auxiliary quadratic programming problem (17) setting the appropriate parameter $R_{k}$. Furthermore, let $g\left(R_{k}\right)=R_{k}-\sqrt{\sum_{j=1}^{n}\left\{y_{k j}^{\left(R_{k}\right)}\right\}^{2}}$. Then, the following theorem to determine that the appropriate value of $R$ is derived.

Theorem 2 (see [26]). Let $\mathbf{y}_{k}^{*}$ be the optimal solution to problem (17) and optimal value $R_{k}^{*}=\sqrt{\sum_{j=1}^{n}\left\{y_{k j}^{*}\right\}^{2}}$. Then the following relationship holds:

$$
\begin{aligned}
& R_{k}^{*}>R_{k} \Longleftrightarrow g\left(R_{k}\right)>0 \\
& R_{k}^{*}=R_{k} \Longleftrightarrow g\left(R_{k}\right)=0 \\
& R_{k}^{*}<R_{k} \Longleftrightarrow g\left(R_{k}\right)<0 .
\end{aligned}
$$

This theorem means that an optimal solution $y_{k j}^{*}$ of auxiliary problem (17) is an optimal and unique solution of main problem (16) in the only case $R_{k}^{*}=R_{k}$.

Lagrange function $L$ of problem (17) is obtained using Lagrange multipliers $\lambda$ and $v_{j}$ as follows:

$$
L=-R_{k} \sum_{j=1}^{n} \bar{r}_{k j}^{\prime} y_{k j}+\frac{d_{k}}{2} \sum_{j=1}^{n} y_{k j}^{2}+\lambda\left(\sum_{j=1}^{n} a_{k j} y_{k j}-1\right)-\sum_{j=1}^{n} v_{j} y_{k j} .
$$

Karush-Kuhn-Tucker condition of this Lagrange function is also calculated as follows:

$$
\begin{gathered}
-R_{k} \bar{r}_{k j}^{\prime}+d_{k} y_{k j}+\lambda a_{k j}-v_{j}=0 \\
\sum_{j=1}^{n} a_{k j} y_{k j}=1 \\
v_{j} y_{k j}=0, \quad(j=1,2, \ldots, n),
\end{gathered}
$$


where $v_{j} y_{k j}=0$ is the complementarity condition. Solving this simultaneous equation, we obtain the following optimal solution of problem (17):

$$
y_{k j}^{\left(R_{k}\right)}= \begin{cases}\frac{R_{k} \bar{r}_{k j}^{\prime}-\lambda a_{k j}}{d_{k}} & \left(\lambda \leq \frac{R_{k} \bar{r}_{k j}^{\prime}}{a_{k j}}\right) \\ 0 & \left(\lambda \geq \frac{R_{k} \bar{r}_{k j}^{\prime}}{a_{k j}}\right) .\end{cases}
$$

This solution $y_{k j}^{\left(R_{k}\right)}$ depends on parameters $R_{k}$ and $\lambda$, and, hence, we need to determine the optimal value of parameters $R_{k}$ and $\lambda$. We define $A_{j}=R_{k} \bar{r}_{k j}^{\prime} / a_{k j}$. With respect to the order of $A_{1}, A_{2}, \ldots, A_{n}$, the following theorem holds.

Theorem 3. The order of $A_{1}, A_{2}, \ldots, A_{n}$ is determined without depending on $R_{k}$.

Proof. With respect to any two parameters $A_{i}$ and $A_{j}$,

$$
A_{i}-A_{j}=\frac{R_{k} \bar{r}_{k i}^{\prime}}{a_{k i}}-\frac{R_{k} \bar{r}_{k j}^{\prime}}{a_{k j}}=R_{k}\left(\frac{\bar{r}_{k i}^{\prime}}{a_{k i}}-\frac{\bar{r}_{k j}^{\prime}}{a_{k j}}\right)
$$

holds. Since $R_{k} \geq 0$, the positive or the negative of $A_{i}-A_{j}$ is determined by the value of $\bar{r}_{k i}^{\prime} / a_{k i}-\bar{r}_{k j}^{\prime} / a_{k j}$ not depending on $R_{k}$.

Consequently, the following exact algorithm is developed to obtain the optimal solution based on the above theorem.

Exact Algorithm for Problem (17).

Step 1. Sort $A_{1}, A_{2}, \ldots, A_{n}$ in ascending order according to values of $\bar{r}_{k j}^{\prime} / a_{k j},(j=1, \ldots, n)$. Go to Step 2 .

Step 2. Set $A_{0} \leftarrow 0$ and $h \leftarrow 1$. Go to Step 3 .

Step 3. Obtain an optimal solution derived from solution (21) depending on $R_{k}$ :

$$
\begin{aligned}
& \sum_{j=h}^{n} a_{k j}\left(\frac{R_{k} \bar{r}_{k j}^{\prime}-\lambda a_{k j}}{d_{k}}\right)=1 \Longleftrightarrow \lambda=\frac{R_{k}\left(\sum_{j=h}^{n} a_{k j} \bar{r}_{k j}^{\prime}\right)-d_{k}}{\sum_{j=h}^{n} a_{k j}^{2}}
\end{aligned}
$$

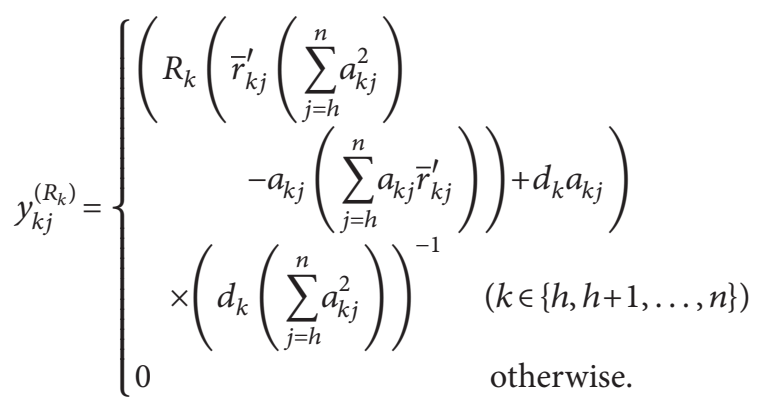

Step 4. Solve the following quadratic equation of $R_{k}^{(h)}$ :

$$
\begin{gathered}
\left\{R_{k}^{(h)}\right\}^{2} \\
=\sum_{j=h}^{n}\left\{\left(R _ { k } \left(\bar{r}_{k j}^{\prime}\left(\sum_{j=h}^{n} a_{k j}^{2}\right)\right.\right.\right. \\
\left.\left.-a_{k j}\left(\sum_{j=h}^{n} a_{k j} \bar{r}_{k j}^{\prime}\right)\right)+d_{k} a_{k j}\right) \\
\left.\times\left(d_{k}\left(\sum_{j=h}^{n} a_{k j}^{2}\right)\right)^{-1}\right\}^{2}
\end{gathered}
$$

and substitute $R_{k}^{(h)}$ into $\lambda^{(h)}=\left(R_{k}\left(\sum_{j=h}^{n} a_{k j} \bar{r}_{k j}^{\prime}\right)-d_{k}\right) / \sum_{j=h}^{n} a_{k j}^{2}$. Go to Step 5.

Step 5. If $\lambda^{(h)}$ satisfies $A_{h-1} \leq \lambda^{(h)} \leq A_{h}, y_{k j}^{\left(R_{k}\right)}$ is the optimal solution of problem (16). Terminates this algorithm. If not, $h \leftarrow h+1$ and return to Step 3 .

Using this algorithm, the optimal solution of problem (12) is explicitly obtained as $\mathbf{x}^{*}=\left(\Lambda_{k}^{1 / 2}\right)^{-1} \mathbf{P}_{k} \mathbf{y}_{k}^{*}$.

3.2. Solution Approach of Our Proposed Model Robust-Based Multiobjective Portfolio Selection Problem. Since investors obtain positive ideal values $z_{d_{k}}^{*}$ and $E^{*}\left(\overline{\mathbf{r}}_{k}^{t} \mathbf{x}\right)$ and minimum target values $z_{d_{k}}^{-}$and $E^{-}\left(\overline{\mathbf{r}}_{k}^{t} \mathbf{x}\right)$ from the above exact algorithm, we next consider the optimal solution of the following reprovided main problem (11):

$$
\begin{aligned}
\text { Minimize } & \sum_{k \in\{C, L, U\}} w_{d_{k}}\left\{\frac{z_{d_{k}}^{*}-z_{d_{k}}}{z_{d_{k}}^{*}-z_{d_{k}}^{-}}\right\} \\
& +\sum_{k \in\{C, L, U\}} w_{E_{k}}\left\{\frac{E^{*}\left(\overline{\mathbf{r}}_{k}^{t} \mathbf{x}\right)-E\left(\overline{\mathbf{r}}_{k}^{t} \mathbf{x}\right)}{E^{*}\left(\overline{\mathbf{r}}_{k}^{t} \mathbf{x}\right)-E^{-}\left(\overline{\mathbf{r}}_{k}^{t} \mathbf{x}\right)}\right\}
\end{aligned}
$$

subject to $\quad \mathbf{1}^{t} \mathbf{x}=1, \quad \mathbf{x} \geq \mathbf{0}$.

In this paper, we assume that variances of $\sigma_{L j}^{2}$ and $\sigma_{U j}^{2}$ are uniformly larger than $\sigma_{C j}^{2}$; that is, $\sigma_{L j}^{2}=\alpha_{L}^{2} \sigma_{C j}^{2}$ and $\sigma_{U j}^{2}=\alpha_{U}^{2} \sigma_{C j}^{2}$ satisfying $\alpha_{L}>1$ and $\alpha_{U}>1$, and also assume that correlation coefficients of $\mathbf{V}_{L}$ and $\mathbf{V}_{U}$ are the same as $\mathbf{V}_{C}$. From these assumptions, we obtain $\mathbf{V}_{L}=\alpha_{L}^{2} \mathbf{V}_{C}$ and $\mathbf{V}_{U}=\alpha_{U}^{2} \mathbf{V}_{C}$. Substituting $\mathbf{V}_{L}$ and $\mathbf{V}_{U}$, problem (25) is equivalently transformed into the following problem:

Minimize $-\sum_{k \in\{C, L, U\}}\left\{\frac{w_{d_{k}}}{z_{d_{k}}^{*}-z_{d_{k}}^{-}}+\frac{w_{E_{k}}}{E^{*}\left(\mathbf{r}_{k}^{t} \mathbf{x}\right)-E^{-}\left(\mathbf{r}_{k}^{t} \mathbf{x}\right)}\right\}$

$$
\times E\left(\mathbf{r}_{k}^{t} \mathbf{x}\right)+\left(\sum_{k \in\{C, L, U\}} \frac{w_{d_{k}} d_{k} \alpha_{k}}{z_{d_{k}}^{*}-z_{d_{k}}^{-}}\right)
$$




$$
\begin{array}{r}
\times \sqrt{\mathbf{V}\left(\mathbf{r}_{k}^{t} \mathbf{x}\right)} \\
\text { subject to } \quad \mathbf{1}^{t} \mathbf{x}=1, \quad \mathbf{x} \geq \mathbf{0},
\end{array}
$$

where $\alpha_{C}=1$. Since $w_{d_{k}} /\left(z_{d_{k}}^{*}-z_{d_{k}}^{-}\right)+w_{E_{k}} /\left(E^{*}\left(\mathbf{r}_{k}^{t} \mathbf{x}\right)-E^{-}\left(\mathbf{r}_{k}^{t} \mathbf{x}\right)\right)$ and $w_{d_{k}} d_{k} \alpha_{k} /\left(z_{d_{k}}^{*}-z_{d_{k}}^{-}\right)$are constant values, this problem is the same as problem (16). Therefore, we can apply the same exact algorithm to problem (26) and obtain the explicit optimal portfolio.

\section{Conclusion}

In this paper, we have proposed a robust-based portfolio selection problem with most probable random distribution derived from current market data and other random distributions of boom and recession. Since the proposed model is a multiobjective programming problem, we have introduced a principle of compromise considering the shortest distance from positive ideal values, called Minkowski's $L p$-metric. In the main problem, we need to set some parameters by solving subproblems, and hence, we have developed the exact algorithm and shown the explicit optimal solution. Furthermore, by introducing a natural assumption for variances to random distributions of boom and recession, the main problem of our proposed model is transformed into a standard existing problem. In our proposed model, robust parameter is flexibly controlled according to investor's risk-averse subjectivity, and, hence, our proposed model will be more useful than previous models in terms of the flexible risk-controlled and support usual and unusual economic conditions.

As for future studies, we need to consider the application of the proposed model and solution algorithm to largescale or multiperiod portfolio selection problems using real market data. Furthermore, it may be appropriate to set robustness parameter $d$ introduced in (4) as a membership function based on the fuzzy theory in terms of realistic modelling, because $d$ is set by investor's risk-averse subjectivity which includes fuzziness. In addition, since the proposed model obtains the explicit optimal portfolio according to the investor's risk-averse subjectivity, we should tackle sensitivity analysis for the parameters of the proposed robust portfolio model. The sensitivity analysis may be useful for economists to estimate how investors' risk-averse subjectivity has an impact on the current market and how to find the appropriate balance between robustness and the total profit in the nearfuture market.

\section{Conflict of Interests}

The authors declare that there is no conflict of interests regarding the publication of this paper.

\section{References}

[1] H. M. Markowitz, "Portfolio selection," The Journal of Finance, vol. 7, no. 1, pp. 77-91, 1952.
[2] H. Konno and H. Yamazaki, "Mean-absolute deviation portfolio optimization model and its applications to Tokyo stock market," Management Science, vol. 37, pp. 519-531, 1991.

[3] H. Konno, H. Shirakawa, and H. Yamazaki, "A mean-absolute deviation-skewness portfolio optimization model," Annals of Operations Research, vol. 45, no. 1-4, pp. 205-220, 1993.

[4] V. S. Bawa and E. B. Lindenberg, "Capital market equilibrium in a mean-lower partial moment framework," Journal of Financial Economics, vol. 5, pp. 189-200, 1977.

[5] E. J. Elton and M. J. Gruber, Modern Portfolio Theory and Investment Analysis, Wiley, New York, NY, USA, 1995.

[6] R. T. Rockafellar and S. Uryasev, "Optimization of conditional value-at-risk," Journal of Risk, vol. 2, no. 3, pp. 1-21, 2000.

[7] A. Bilbao-Terol, B. Pérez-Gladish, M. Arenas-Parra, and M. V. Rodríguez-Uría, "Fuzzy compromise programming for portfolio selection," Applied Mathematics and Computation, vol. 173, no. 1, pp. 251-264, 2006.

[8] C. Carlsson, R. Fullér, and P. Majlender, "A possibilistic approach to selecting portfolios with highest utility score," Fuzzy Sets and Systems, vol. 131, no. 1, pp. 13-21, 2002.

[9] L. Duan and P. Stahlecker, "A portfolio selection model using fuzzy returns," Fuzzy Optimization and Decision Making, vol. 10, no. 2, pp. 167-191, 2011.

[10] X. Huang, "Fuzzy chance-constrained portfolio selection," Applied Mathematics and Computation, vol. 177, no. 2, pp. 500507, 2006.

[11] X. Huang, "A review of credibilistic portfolio selection," Fuzzy Optimization and Decision Making, vol. 8, no. 3, pp. 263-281, 2009.

[12] M. Inuiguchi and T. Tanino, "Portfolio selection under independent possibilistic information," Fuzzy Sets and Systems, vol. 115, no. 1, pp. 83-92, 2000.

[13] H. Tanaka and P. Guo, "Portfolio selection based on upper and lower exponential possibility distributions," European Journal of Operational Research, vol. 114, pp. 115-126, 1999.

[14] H. Tanaka, P. Guo, and I. B. Türksen, "Portfolio selection based on fuzzy probabilities and possibility distributions," Fuzzy Sets and Systems, vol. 111, no. 3, pp. 387-397, 2000.

[15] J. Watada, "Fuzzy portfolio selection and its applications to decision making," Tatra Mountains Mathematical Publications, vol. 13, pp. 219-248, 1997.

[16] H. Katagiri, M. Sakawa, and H. Ishii, "A study on fuzzy random portfolio selection problems using possibility and necessity measures," Scientiae Mathematicae Japonicae, vol. 65, no. 2, pp. 361-369, 2005

[17] T. Hasuike, H. Katagiri, and H. Ishii, "Portfolio selection problems with random fuzzy variable returns," Fuzzy Sets and Systems, vol. 160, no. 18, pp. 2579-2596, 2009.

[18] X. Huang, "Two new models for portfolio selection with stochastic returns taking fuzzy information," European Journal of Operational Research, vol. 180, no. 1, pp. 396-405, 2007.

[19] T. Hasuike and H. Ishii, "Portfolio selection problem with two possibilities of the expected return," in Proceedings of the 4th International Conference on Nonlinear Analysis and Convex Analysis, pp. 115-125, Yokohama Publishers, 2005.

[20] D. Goldfarb and G. Iyengar, "Robust portfolio selection problems," Mathematics of Operations Research, vol. 28, no. 1, pp. 138, 2003.

[21] C. Gregory, K. Darby-Dowman, and G. Mitra, "Robust optimization and portfolio selection: the cost of robustness," European Journal of Operational Research, vol. 212, no. 2, pp. 417-428, 2011. 
[22] J. Liesiö, P. Mild, and A. Salo, "Robust portfolio modeling with incomplete cost information and project interdependencies," European Journal of Operational Research, vol. 190, no. 3, pp. 679-695, 2008.

[23] M. S. Lobo, Robust and convex optimization with applications in finance [Doctor thesis], Department of Electrical Engineering and the Committee on Graduate Studies, Stanford University, 2000.

[24] A. Ben-Tal and A. Nemirovski, "Robust solutions of uncertain linear programs," Operations Research Letters, vol. 25, no. 1, pp. $1-13,1999$.

[25] A. Ben-Tal and A. Nemirovski, Lectures on Modern Convex Optimization, Society for Industrial and Applied Mathematics (SIAM), Philadelphia, Pa, USA, 2001.

[26] T. Hasuike and H. Katagiri, "Robust-based interactive portfolio selection problems with an uncertainty set of returns," Fuzzy Optimization and Decision Making, vol. 12, no. 3, pp. 263-288, 2013. 


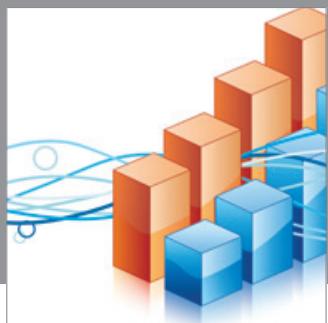

Advances in

Operations Research

mansans

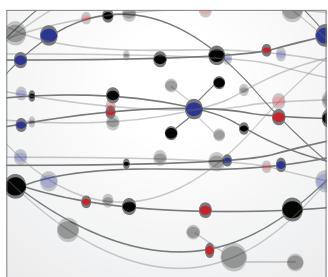

The Scientific World Journal
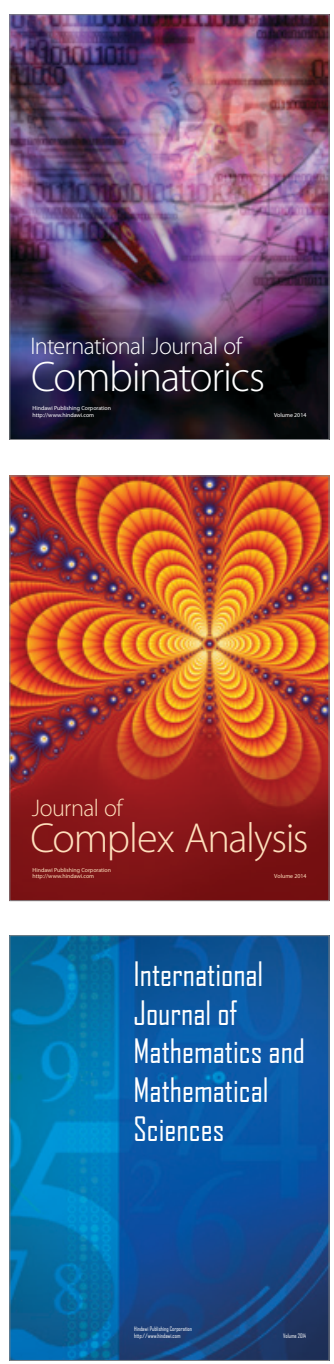
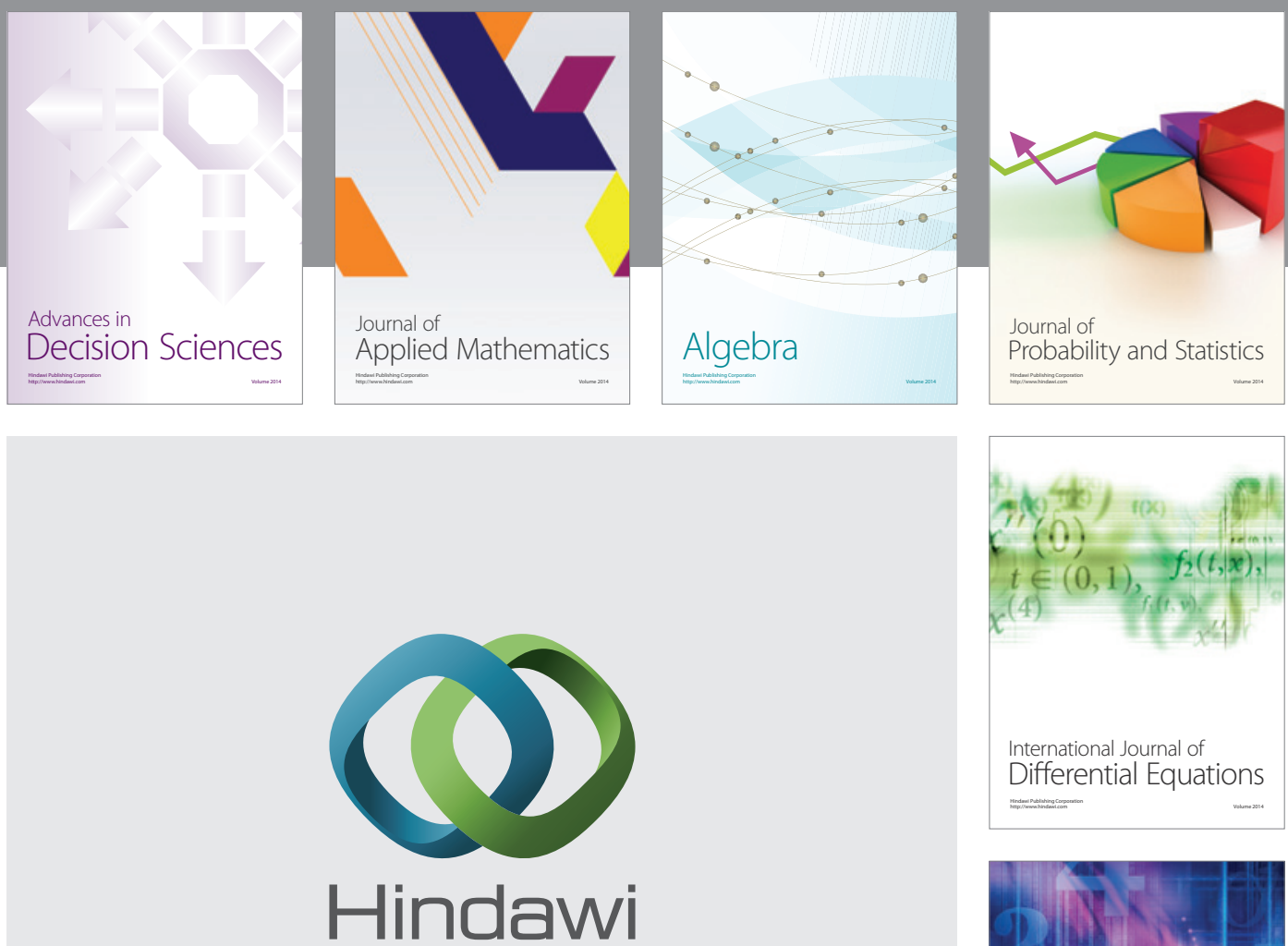

Submit your manuscripts at http://www.hindawi.com
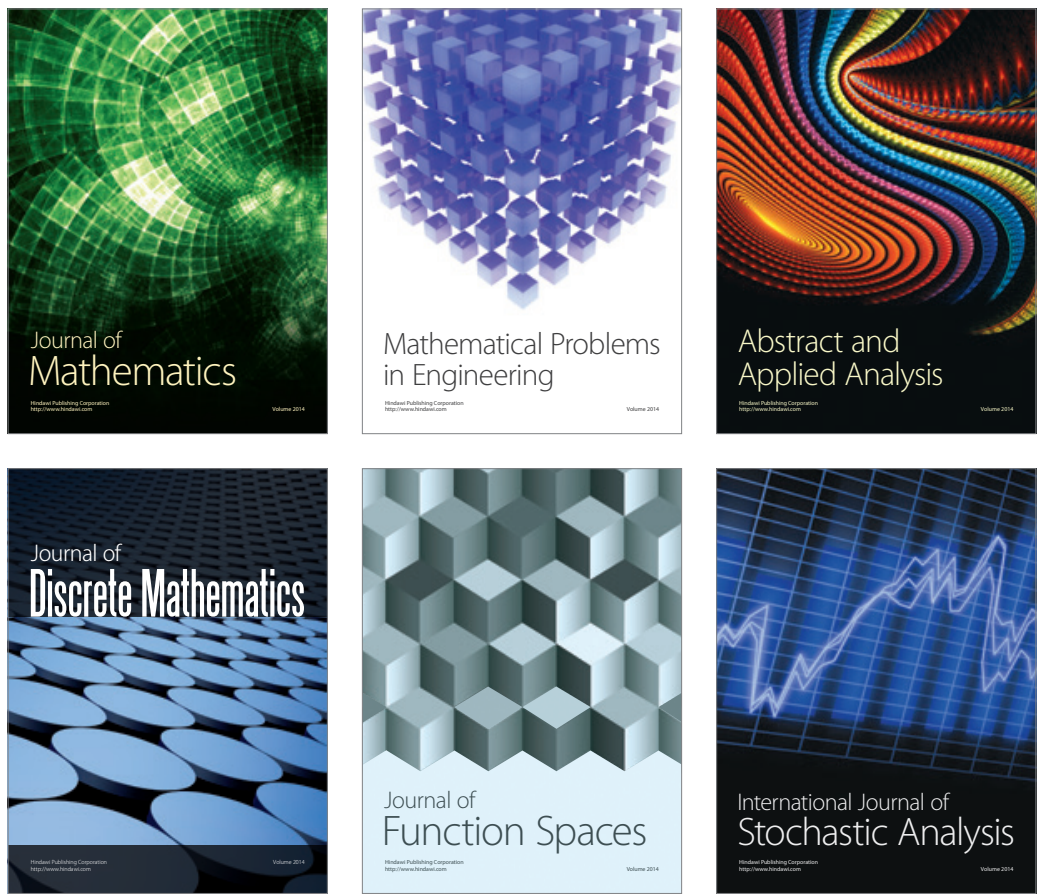

Journal of

Function Spaces

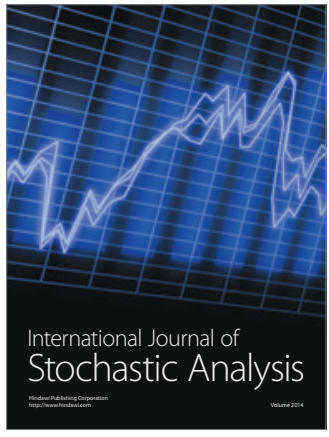

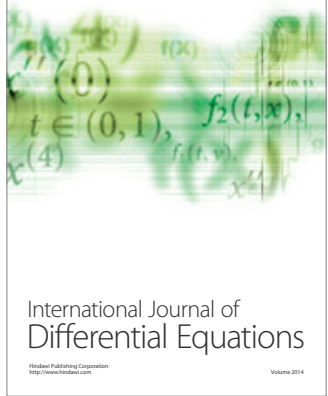
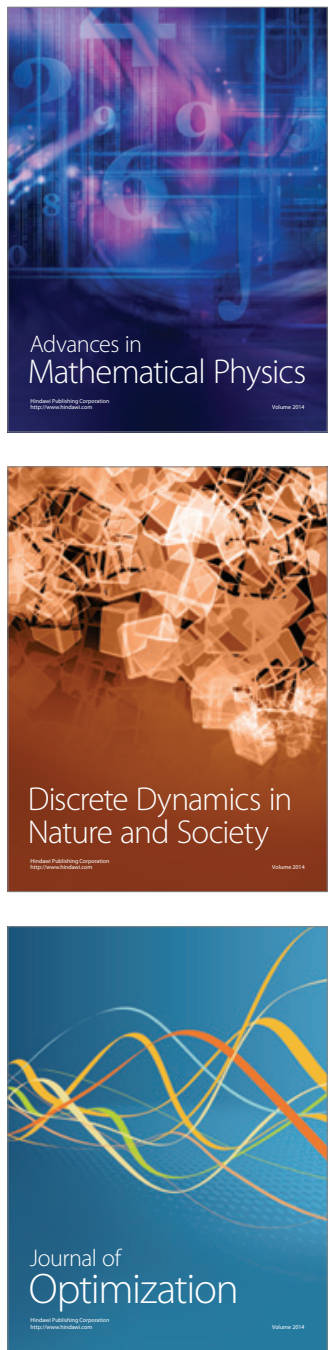